\title{
1 Discovery of a 382-nt deletion during the early evolution of SARS-CoV-2
}

2

3 Running title: A SARS-CoV-2 deletion variant

4

5 Yvonne CF Su ${ }^{1 \dagger}$, Danielle E Anderson ${ }^{1 \dagger}$, Barnaby E Young ${ }^{2,3,9 \ddagger}$, Feng Zhu ${ }^{1 \dagger}$, Martin

6 Linster ${ }^{1 \ddagger}$, Shirin Kalimuddin ${ }^{1,4}$, Jenny GH Low ${ }^{1,4}$, Zhuang Yan ${ }^{1}$, Jayanthi Jayakumar ${ }^{1}$, Louisa

$7 \quad$ Sun $^{5}$, Gabriel Z Yan ${ }^{6}$, Ian H Mendenhall ${ }^{1}$, Yee-Sin Leo ${ }^{2,3,7,8,9}$, David Chien Lye ${ }^{2,3,8,9}$, Lin-Fa

8 Wang $^{1,10^{*}}$, Gavin JD Smith ${ }^{1,10^{*}}$

9

$10{ }^{1}$ Programme in Emerging Infectious Diseases, Duke-NUS Medical School; ${ }^{2}$ National Centre

11 for Infectious Diseases; ${ }^{3}$ Tan Tock Seng Hospital; ${ }^{4}$ Singapore General Hospital; ${ }^{5}$ Alexandra

12 Hospital; ${ }^{6}$ National University Hospital; ${ }^{7}$ Saw Swee Hock School of Public Health, ${ }^{8}$ Yong

13 Loo Lin School of Medicine, ${ }^{9}$ Lee Kong Chian School of Medicine, ${ }^{10}$ SingHealth Duke-NUS

14 Global Health Institute, Singapore

15

16 These authors contributed equally

17 \$These authors contributed equally

18 *To whom correspondence may be addressed. LFW linfa.wang@,duke-nus.edu.sg and GJDS

19 gavin.smith@duke-nus.edu.sg. 
To date, the SARS-CoV-2 genome has been considered genetically more stable than SARS-CoV or MERS-CoV. Here we report a 382-nt deletion covering almost the entire open reading frame 8 (ORF8) of SARS-CoV-2 obtained from eight hospitalized patients in Singapore. The deletion also removes the ORF8 transcription-regulatory sequence (TRS), which in turn enhances the downstream transcription of the $\mathbf{N}$ gene. We also found that viruses with the deletion have been circulating for at least four weeks. During the SARS-CoV outbreak in 2003, a number of genetic variants were observed in the human population [1], and similar variation has since been observed across SARSrelated CoVs in humans and bats. Overwhelmingly these viruses had mutations or deletions in ORF8, that have been associated with reduced replicative fitness of the virus [2]. This is also consistent with the observation that towards the end of the outbreak sequences obtained from human SARS cases possessed an ORF8 deletion that may be associated with host adaptation [1]. We therefore hypothesise that the major deletion revealed in this study may lead to an attenuated phenotype of SARS-CoV-2.

On 1 December 2019, a novel coronavirus emerged from Hubei province in China and infected people visiting the Huanan seafood market in Wuhan [3]. The virus demonstrated efficient human-to-human transmission within mainland China and subsequently spread across many countries. The virus was soon identified as 2019-nCoV, more recently designated SARS-CoV-2, while the disease is referred to as COVID-19. On 30 January 2020, the World Health Organization declared a Public Health Emergency of International Concern. As of 10 March 2020, the COVID-19 outbreak has led to 113,702 confirmed cases and 4,012 deaths globally [4]. Zoonotic viruses have crossed the species barrier from animals to humans and the success of an interspecies transmission is the ability of a novel virus to 
adapt, via genetic mutation events, to a new host and cause sustained transmission that can lead to a significant outbreak.

Nasopharyngeal swabs collected from hospitalized patients positive for SARS-CoV-2 in Singapore were subjected to next generation sequencing (NGS) analysis, with and without passaging in Vero-E6 cells. The NGS data revealed a 382-nt deletion towards the 3' end of the viral genomes obtained from multiple patients (Suppl. Table 1). To confirm this observation, specific PCR primers were designed flanking the deleted region and Sanger sequencing performed (Suppl. Fig. 1). This verified the deletion at positions 27,848 to 28,229 of the SARS-CoV-2 genome. Interrogation of the NGS assemblies of these 382-nt deletion variants (referred to hereafter as $\Delta 382$ ) indicated that the virus populations were homogenous.

Apart from the 382-nt deletion, the genome organization of the $\Delta 382$ viruses is identical to that of other SARS-CoV-2 (Fig. 1A). Closer examination of the deletion indicated that it spans an area of the ORF7b and ORF8 that includes the ORF8 transcriptional regulator sequence (TRS), eliminating ORF8 transcription (Fig. 1B). The ORF8 region has been identified as an evolutionary hotspot of SARSr-CoVs. For instance, sequences of human SARS-CoV TOR2 and LC2 exhibit 29-nt and 415-nt deletions, whereas those of bat SARSCoV JTMC15 have discontinuous deletions in ORF7/8 (Fig. 1B) [5-8]. The ORF8 region of SARS-CoVs has been shown to play a significant role in adaptation to the human host following interspecies transmission [7] and virus replicative efficiency [2]. Similarly the disruption of ORF8 region in $\Delta 382$ viruses may be a result of human adaptation after the emergence of SARS-CoV-2. 
69 To investigate the possible effects of the TRS deletion, we calculated and compared the total number of transcripts per million (TPM) of each gene between wild-type (WT) and $\Delta 382$

71 viruses. For each gene, we counted unambiguous reads that uniquely mapped to the genespecific transcripts that include the joint leader and TRS sequences [9]. We analysed three WT and two $\Delta 382$ sequences for which two independent NGS library preparations were available. Our results indicate differential patterns of TPM level between WT and $\Delta 382$ viruses (Fig. 2A). The $\triangle 382$ sequences displayed a greater level of TPMs in the ORF6, ORF7a and N genes compared to WT. More specifically, the $\Delta 382 \mathrm{~N}$ gene, downstream of ORF8, showed a significant increase in the TPM level. To further determine the impact of the 382-nt deletion on the transcription of the $\mathrm{N}$ gene, we conducted qPCR to measure the relative abundance of the RNAs from 2 genes $(\mathrm{E}$ and $\mathrm{N})$ located upstream and downstream of the deleted region, respectively. Results shown in Figure 2B confirm the enhancement of $\mathrm{N}$ gene transcription in $\Delta 382$ viruses. This enhancement was consistently observed regardless of the sample type, whether directly from swab samples or passaged viruses.

The global phylogeny of SARS-CoV-2 (Suppl. Fig. 2) revealed a comb-like appearance in the phylogenetic tree and a general lack of phylogenetic resolution, reflecting the high similarity of the virus genomes. This tree topology is a typical characteristic of a novel interspecies transmission, wherein the viruses are infecting immunologically naïve populations with sustained human-to-human transmission, as previously shown for the early stages of the $2009 \mathrm{H} 1 \mathrm{~N} 1$ pandemic [10]. Notably, we observe an emerging virus lineage (tentatively designated as "Lineage 1" in Suppl. Fig. 2) that consists of sequences from various newly affected countries with recent virus introductions. All viruses from this recent 
gene [11], which is known to undergo strong positive selection during animal-to-human transmission [7].

To estimate the divergence times among lineages of SARS-CoV-2, we reconstructed a dated phylogeny based on 137 complete genomes, including data from this study. The estimated rate of nucleotide substitutions among SARS-CoV-2 viruses is approximately at $8.68 \times 10^{-4}$ substitutions per site per year (95\% HPD: $\left.5.44 \times 10^{-4}-1.22 \times 10^{-3}\right)$, which is moderately lower than SARS-CoV ([12]: $\left.0.80-2.38 \times 10^{-3}\right)$ and MERS-CoV (with a mean rate [13] of: $1.12 \times 10^{-3}$ and 95\% HPD: $58.76 \times 10^{-4}-1.37 \times 10^{-3}$ ) as well as human seasonal influenza viruses (ranging $1.0-5.5 \times 10^{-3}$ depending on individual gene segments $[10,14-16]$. In contrast, the estimated rate of SARS-CoV-2 viruses is greater than estimates for human coronaviruses by a magnitude order of $4 \times 10^{-4}[17]$.

The mean TMRCA estimates indicate the introduction of SARS-CoV-2 into humans (Fig. 3. node A) occurred in the middle of November 2019 (95\% HPD: 2019.77-2019.94) (Table 1), suggesting that the viruses were present in human hosts approximately one month before the outbreak was detected. A single amino-acid mutation was fixed on the ORF8 region of all full-genome Lineage 1 viruses (Fig. 3, node B), with the TMRCA estimate of 22 December 2019 (95\% HPD: 2019.9-2020.0). The $\Delta 382$ viruses form a monophyletic group within Lineage 1, and dating estimates indicate that they may have arisen in the human population in Singapore around 07 February 2020 (95\% HPD: 2020.12-2020.06), consistent with the date of collection of the $\Delta 382$ positive samples (17-19 February 2020). The three $\Delta 382$ viruses shared a high level of nucleotide similarities (99.9\%) with only 5 nucleotide differences between them. Although the dated phylogeny indicates that $\Delta 382$ viruses clustered together and are possibly derived from a single source, there is a general lack of statistical support in 
118 SARS-CoV-2 phylogenies due to its recent emergence, and evolutionary inferences should be

119 made with caution.

120

121 In this report we describe the first major evolutionary event of the SARS-CoV-2 virus

122 following its emergence into the human population. Although the biological consequences of

123 this deletion remain unknown, the alteration of the $\mathrm{N}$ gene transcription would suggest this

124 may have an impact on virus phenotype. Recent work has indicated that ORF8 of SARS-CoV

125 plays a functional role in virus replicative fitness and may be associated with attenuation

126 during the early stages of human-to-human transmission [2]. Given the prevalence of a

127 variety of deletions in the ORF8 of SARSr-CoVs, it is likely that we will see further deletion

128 variants emerge with the sustained transmission of SARS-CoV-2 in humans. Future research

129 should focus on the phenotypic consequences of $\Delta 382$ viruses in the transmission dynamics

130 of the current epidemics and the immediate application of this genomic marker for molecular

131 epidemiological investigation. 
132

133

134

135

136

137

138

139

140

141

142

143

144

145

146

147

148

149

150

151

152

153

154

155

Online content. Any methods, additional references, Nature Research reporting summaries, source data, extended data, supplementary information, acknowledgements, peer review information; details of author contributions and competing interests; and statements of data availability are available at [Article DOI].

Data availability. The new sequences generated in this study have been deposited in GISAID database under accession numbers EPI_ISL_407987, EPI_ISL_407988, EPI_ISL_410535 to EPI_ISL_410537 for WT viruses and EPI_ISL_414378 to EPI_ISL_414380 for $\Delta 382$ viruses.

Acknowledgements. This study was supported by the Duke-NUS Signature Research Programme funded by the Ministry of Health, Singapore, the National Medical Research Council under its COVID-19 Research Fund (NMRC Project No. COVID19RF-001) and by National Research Foundation Singapore grant NRF2016NRFNSFC002-013 (Combating the Next SARS-or MERS-Like Emerging Infectious Disease Outbreak by Improving Active Surveillance). We thank Viji Vijayan, Benson Ng and Velraj Sivalingam of the Duke-NUS Medical School ABSL3 facility for logistics management and assistance. We thank all scientific staff who assisted with processing clinical samples, especially Velraj Sivalingam, Adrian Kang, Randy Foo, Wan Ni Chia and Akshamal Gamage. We thank Su Ting Tay, Ming Hui Lee and Angie Tan from the Duke-NUS Medical School Genome Biology Facility for expert and rapid technical assistance.

Author contributions. YCFS, DEA, LFW, GJDS designed and supervised research. BEY, SK, JGHL, LS, GZY, YSL, DCL collected and provided samples. DEA, ML, YZ conducted 
experiments. YCFS, ZF, JJ, IHM, GJDS performed analyses. YCFS, ZF, ML, LFW, GJDS wrote the paper. All authors reviewed and approved the manuscript.

\section{Methods}

Ethics statement. This study was undertaken as part of the national disease outbreak and the response and the protocols were approved by the ethics committee of the National Healthcare Group. Patient samples were collected under PROTECT (2012/00917), a multi-centred Prospective Study to Detect Novel Pathogens and Characterize Emerging Infections. Work undertaken at the Duke-NUS Medical School ABSL3 laboratory was approved by the DukeNUS ABSL3 Biosafety Committee, National University of Singapore and Ministry of Health Singapore.

Virus culture, RNA extraction and sequencing. Clinical samples from infected patients were collected from public hospitals in Singapore from January-February 2020. Material from clinical samples was used to inoculate Vero-E6 cells (ATCC ${ }^{\circledR}$ CRL-1586 ${ }^{\mathrm{TM}}$ ). Total RNA was extracted using E.Z.N.A. Total RNA Kit I (Omega Bio-tek) according to manufacturer's instructions and samples analysed by real-time quantitative reverse transcription-PCR (RTqPCR) for the detection of SARS-CoV-2 as previously described [18]. Whole genome sequencing was performed using next-generation sequencing (NGS) methodology. The cDNA libraries were constructed using TruSeq RNA Library Prep Kit (Illumina) according to the manufacturer's instructions and sequenced on an Illumina MiSeq System. Raw NGS reads were trimmed by Trimmomatic v0.39 [19] to remove adaptors and low-quality bases. Genome sequences were assembled and consensus sequences obtained using the BWA algorithm in UGENE v.33. To verify the presence of the deletion in the SARS-CoV-2 genome, we designed two specific PCR primers (F primer: 5'- 
TGTTAGAGGTACAACAGTACTTT-3' and R primer: 5'-

GGTAGTAGAAATACCATCTTGGA-3') targeting the ORF7-8 regions. For samples with $95^{\circ} \mathrm{C}$ for $1 \mathrm{~min}, 52^{\circ} \mathrm{C}$ for $30 \mathrm{sec}, 72^{\circ} \mathrm{C}$ for $1 \mathrm{~min}$ and a final extension at $72^{\circ} \mathrm{C}$ for $10 \mathrm{~min}$ in a thermal cycler (Applied Biosystems Veriti). Deletions in the PCR products were visualized by gel electrophoresis and confirmed by Sanger sequencing. Three full $\Delta 382$ genomes were generated and designated BetaCoV/Singapore/12/2020, BetaCoV/Singapore/13/2020 and

Genomic characterization. To characterize and map the deletion regions of SARS-CoV-2 viruses, we compared with available SARS-CoV-2 and SARs-CoV related genomes from bat (accession number: AY274119) and LC2 (accession number: AY394999). The viral genome organizations of Wuhan-Hu-1 (accession number: MN908947) and Singapore SARS-CoV-2 (Singapore/2 /2020: EPI_ISL_407987) comprise the following gene order and lengths: 
205 To understand possible effects of the TRS deletion on the ORF8 region, we analysed the total number of transcripts per million (TPM) of each gene between wildtype and $\Delta 382$ variants.

207 For each gene, unambiguous reads which uniquely mapped to the specific joint leader and

208 TRS sequences are counted. We analysed three patients of SARS-CoV-2 wild type and two 209 patients of SARS-CoV-2 variants and independent NGS library preparations were performed 210 twice for each sample. The Wuhan-Hu-1 was used as the reference sequence for genomic 211 coordinates. Leader RNA sequence is situated at the 5' UTR region of the genome and the core sequence consists of 26 nt: UCUCUAAACGAACUUUAAAAUCUGUG. Each of the coding genes is preceded by a core transcription regulatory sequence (TRS) which is highly conserved (i.e. ACGAAC), resembling the leader core sequence. The leader RNA is joined to the TRS regions of different genes and the joining is required for driving subgenomic transcription. Coding sequence (CDS), leader and TRS sequences annotations were generated in Geneious and followed published SARS-CoV studies [9]. To characterize the differential levels of TRS for each gene, $70 \mathrm{nt}$ leader sequence and $230 \mathrm{nt}$ downstream of each TRS sequence were annotated individually to form a $300 \mathrm{bp}$ leader-TRS transcript for the splicingaware aligners in R package rtracklayer ( $\mathrm{v}$ 1.44). NGS raw fastq reads were then mapped to the reference genome by Geneious RNA-Seq mapper with the annotation of splice-junctions for the leader-TRS. For each gene, the TPM of leader-TRS was then calculated in Geneious by excluding ambiguous reads which may come from other TRS. The resulting TPM data was generated in Geneious and graphs plotted using ggplot 2 (v3.2.0) in R v3.6.1. Wilcoxon one-sided tests were performed in R to test the significant differences between WT and $\Delta 382$ samples. We also compared individual $\mathrm{Ct}$ values of $\mathrm{E}$ and $\mathrm{N}$ genes between WT and $\Delta 382$

227 viruses using qRT-PCR assays as previously described [18]. Samples used in this analysis included nasopharyngeal swabs from three WT patient samples and both nasopharyngeal 
swabs and passaged samples from two $\Delta 382$ patients $(n=7)$. Significance between groups was assessed using a one-way analysis of variance (ANOVA) in PRISM v8.3.1.

231

232 Phylogenetic analyses. All available genomes of SARS-CoV-2 with associated virus

233 sampling dates were downloaded from the GISAID database. Genome sequence alignment was performed and preliminary maximum likelihood phylogenies of complete genome were reconstructed using RAxML with 1,000 bootstrap replicates in Geneious R9.0.3 software (Biomatters Ltd). Any sequence outliers were removed from subsequent analyses. To reconstruct a time-scaled phylogeny, an uncorrelated lognormal relaxed-clock model with an exponential growth coalescent prior and the HKY85+ $\Gamma$ substitution model was used in the program BEAST v1.10.4 [20] to simultaneously estimate phylogeny, divergence times and rates of nucleotide substitution. Four independent Markov Chain Monte Carlo (MCMC) runs

241 of 100 million generations were performed and sampled every 10,000 generations. The runs

242 were checked for convergence in Tracer v1.7 [21] and that effective sampling size (ESS)

243 values of all parameters was $>200$. The resulting log and tree files were combined after

244 removing appropriate burn-in values using LogCombiner [20], and the maximum clade credibility (MCC) tree was subsequently generated using TreeAnnontator [20]. 


\section{References:}

247 1. Chinese, S.M.E.C., Molecular evolution of the SARS coronavirus during the course of the SARS epidemic in China. Science, 2004. 303(5664): p. 1666-9.

2. $\quad$ Muth, D., et al., Attenuation of replication by a 29 nucleotide deletion in SARScoronavirus acquired during the early stages of human-to-human transmission. Sci Rep, 2018. 8(1): p. 15177.

3. Lu, R., et al., Genomic characterisation and epidemiology of 2019 novel coronavirus: implications for virus origins and receptor binding. The Lancet, 2020. 395(10224): p. $565-574$.

4. World_Health_Organization. Coronavirus disease (COVID-2019) situation reports. 2020 [cited 2020 Mar 2]; Available from:

5. Chinese, S.M.E.C., Molecular evolution of the SARS coronavirus during the course of the SARS epidemic in China. Science (New York, N.Y.), 2004. 303(5664): p. 16661669.

6. Wang, L.-F., et al., Review of bats and SARS. Emerging infectious diseases, 2006. 12(12): p. 1834-1840.

7. Lau, S.K., et al., Severe Acute Respiratory Syndrome (SARS) Coronavirus ORF8 Protein Is Acquired from SARS-Related Coronavirus from Greater Horseshoe Bats through Recombination. J Virol, 2015. 89(20): p. 10532-47.

8. $\mathrm{Xu}, \mathrm{L}$., et al., Detection and characterization of diverse alpha- and betacoronaviruses from bats in China. Virol Sin, 2016. 31(1): p. 69-77.

9. Hussain, S., et al., Identification of novel subgenomic RNAs and noncanonical Virol, 2005. 79(9): p. 5288-95. 
10. Su, Y.C.F., et al., Phylodynamics of H1N1/2009 influenza reveals the transition from host adaptation to immune-driven selection. Nature Communications, 2015. 6: p. 7952.

11. Tang, X., et al., On the origin and continuing evolution of SARS-CoV-2. National Science Review, 2020.

12. Zhao, Z., et al., Moderate mutation rate in the SARS coronavirus genome and its implications. BMC Evolutionary Biology, 2004. 4(1): p. 21.

13. Cotten, M., et al., Spread, circulation, and evolution of the Middle East respiratory syndrome coronavirus. mBio, 2014. 5(1): p. e01062-13.

14. Vijaykrishna, D., et al., The contrasting phylodynamics of human influenza B viruses. eLife, 2015. 4: p. e05055.

15. Virk, R.K., et al., Divergent evolutionary trajectories of influenza B viruses underlie their contemporaneous epidemic activity. Proceedings of the National Academy of Sciences, 2020. 117(1): p. 619-628.

16. Rambaut, A., et al., The genomic and epidemiological dynamics of human influenza A virus. Nature, 2008. 453(7195): p. 615-619.

17. Vijgen, L., et al., Complete Genomic Sequence of Human Coronavirus OC43: Molecular Clock Analysis Suggests a Relatively Recent Zoonotic Coronavirus Transmission Event. Journal of Virology, 2005. 79(3): p. 1595-1604.

18. Corman, V.M., et al., Detection of 2019 novel coronavirus (2019-nCoV) by real-time RT-PCR. Eurosurveillance, 2020. 25(3): p. 2000045.

19. Bolger, A.M., M. Lohse, and B. Usadel, Trimmomatic: a flexible trimmer for Illumina sequence data. Bioinformatics, 2014. 30(15): p. 2114-2120.

20. Suchard, M.A., et al., Bayesian phylogenetic and phylodynamic data integration using BEAST 1.10. Virus Evol, 2018. 4(1): p. vey016. 
bioRxiv preprint doi: https://doi.org/10.1101/2020.03.11.987222; this version posted March 12, 2020. The copyright holder for this preprint (which was not certified by peer review) is the author/funder. All rights reserved. No reuse allowed without permission.

296 21. Rambaut, A., et al., Posterior Summarization in Bayesian Phylogenetics Using Tracer 1.7. Syst Biol, 2018. 67(5): p. 901-904.

298 
299 Table 1. TMRCA estimates of major SARS-CoV-2 nodes.

\begin{tabular}{cccc}
\hline Node & Mean TMRCA & Lower 95\% HPD & Upper 95\% HPD \\
\hline A & 2019.87 (15 November 2019) & 2019.77 (09 October 2019) & 2019.94 (11 December 2019) \\
B & 2019.97 (22 Dec 2019) & 2019.90 (26 November 2019) & 2020.00 (01 January 2020) \\
C & 2020.09 (07 Feb 2020) & 2020.06 (24 January 2020) & 2020.12 (14 Feb 2020)
\end{tabular}

300 Abbreviations: TMRCA, time to most recent common ancestor, HPD: highest posterior 301 density. 
Figure 1.

A

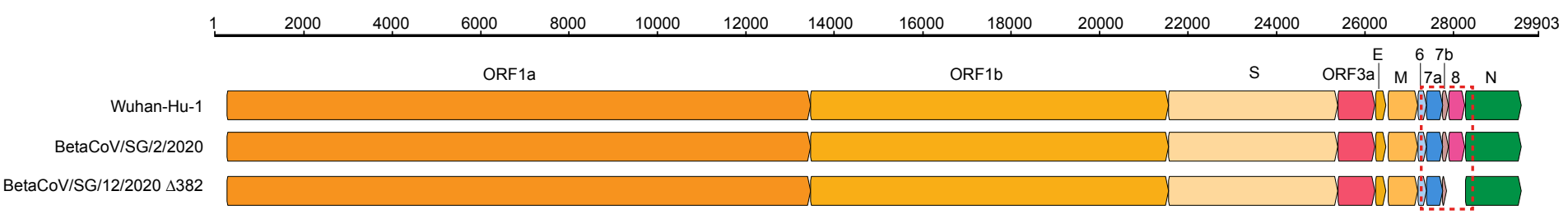

B

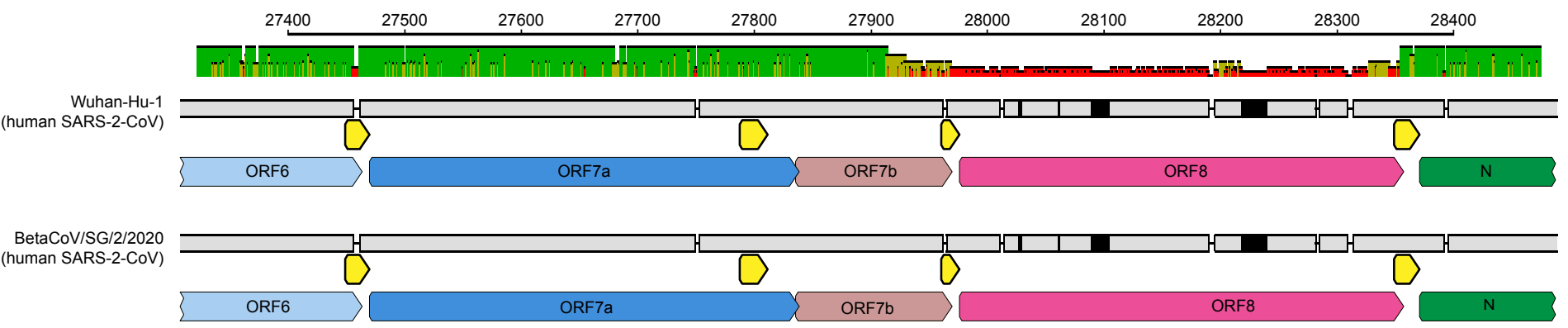

BetaCoV/SG/12/2020 $\Delta 382$ (human SARS-2-CoV)

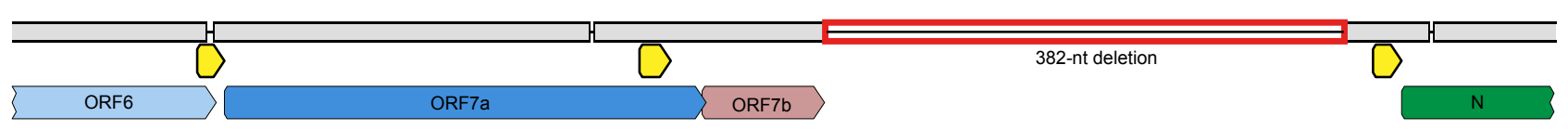

TOR2
(early human SARS-CoV)

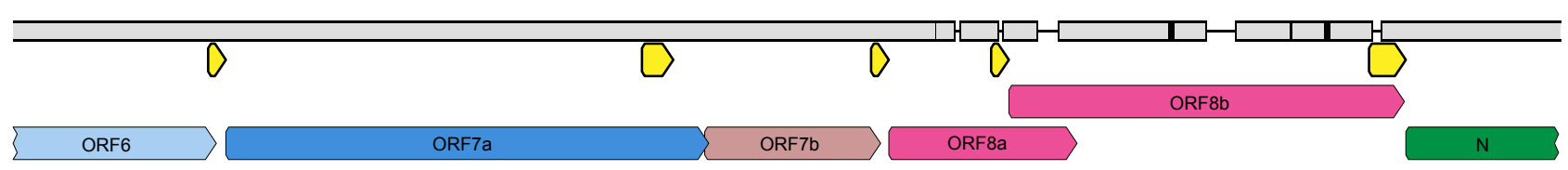

LC2

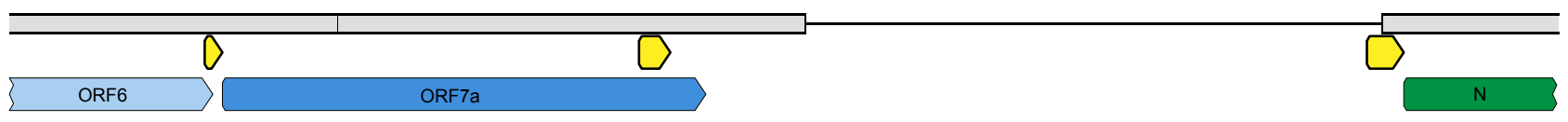

JTMC15
(SARS-CoV-related bat virus)

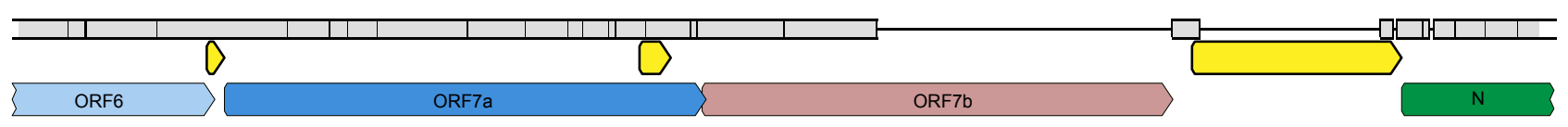

Figure 1. Schematic comparison of SARS-2-CoV, SARS-CoV and SARSr-CoV genomes.

A. Full genome sequences of SARS-2-CoV isolates Wuhan-Hu-1 (GenBank: MN908947), BetaCoV/Singapore/2/2020 (GISAID: 407987), and BetaCoV/Singapore/12/2020 $\Delta 382$ (GISAID: EPI_ISL_414378). B. Magnification of genomic region (dashed box in Figure 1A), numbers on horizontal axes indicate the nucleotide position relative to Wuhan-Hu-1, grey boxes indicate sequence coverage at a certain position, black horizontal lines represent a deletion at a certain position, open reading frames (ORFs) are indicated by colored arrows. A red-lined box indicates the 382-nt deletion. Transcription-regulatory sequences (TRSs) are indicated by yellow arrows. 
Figure $2 \mathrm{~A}$

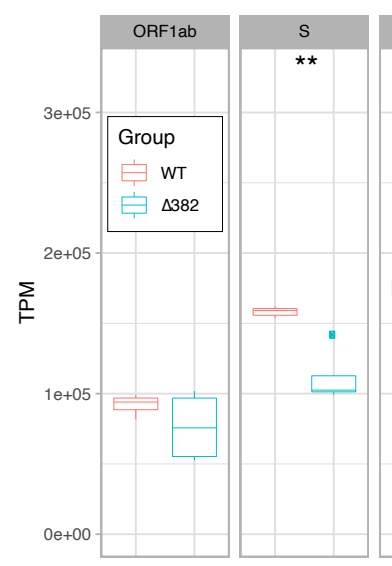

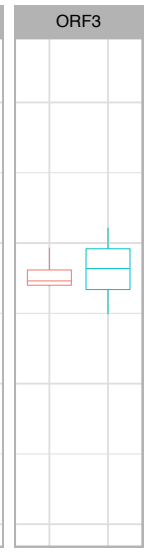

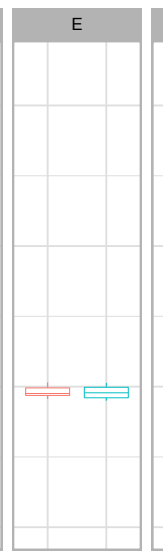

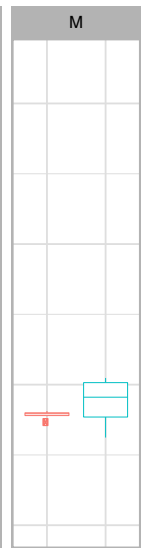

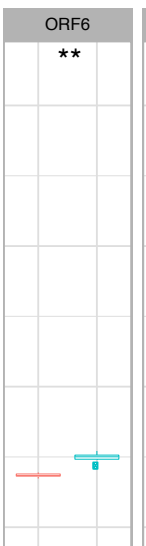

Figure 2B

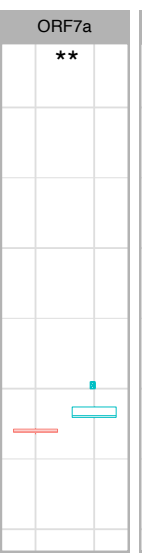

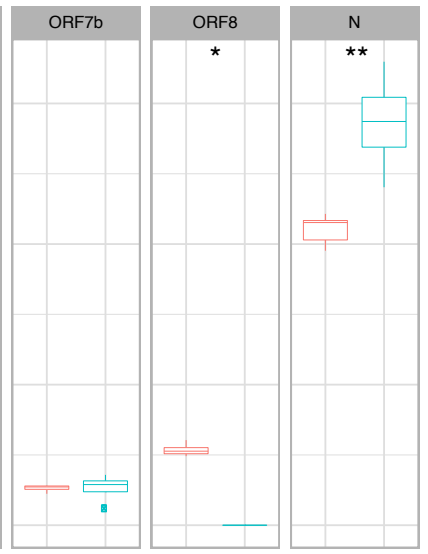

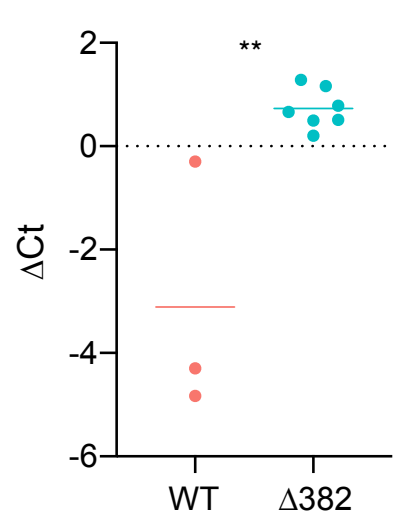

Figure 2. Comparison of transcription of the transcription of the $\mathbf{N}$ gene in wild-type (WT) versus $\Delta 382$ viruses. A. Abundance of mapped reads relative to transcriptional regulatory sequence (TRS) positions across the genome. $70 \mathrm{bp}$ of leader sequence and 230 of each TRS-downstream sequence were merged in a splice-junction in the annotation. Transcripts per million reads (TPM) was calculated from reads mapped specifically to each leader-TRS region and a whisker and scatter plot was drawn for each gene. A Wilcoxon test was applied to the TPM for each gene of $\Delta 382$ to WT $\left(*: p \leq 0.05{ }^{* *}: p \leq 0.01\right)$. B. Relative abundance of the RNAs of the $\mathrm{E}$ gene in comparison to the $\mathrm{N}$ gene of WT versus $\Delta 382$ SARS-CoV-2 ( $p=0.002)$ using qPCR. Horizontal bars indicate the mean of each group. 


\section{Figure 3.}

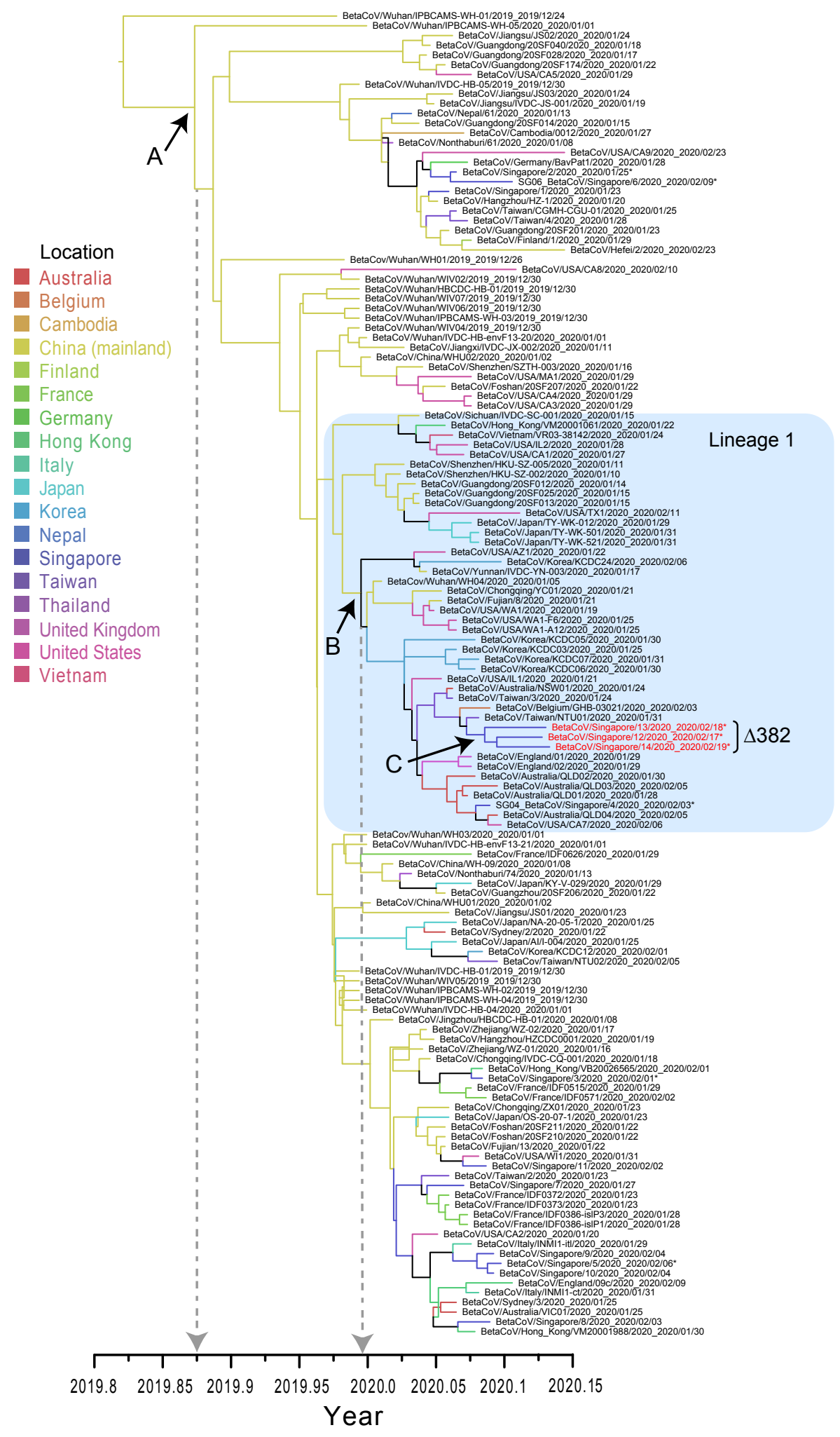

Figure 3. Temporal phylogeny of the complete genomes of SARS-CoV-2 viruses reconstructed using an uncorrelated lognormal relaxed clock model in BEAST. Isolate names in red indicate $\Delta 382$ viruses with a large deletion in the ORF7/8 region as described in this study. ${ }^{*}$ Indicates virus genomes generated in this study. Colored branches denote different geographical locations. 
12.

man

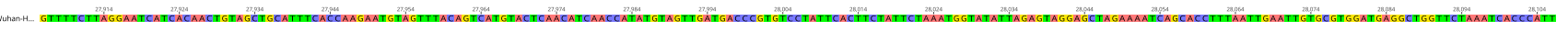

$\left(x_{10}\right.$

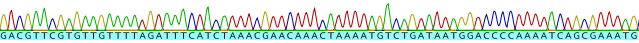

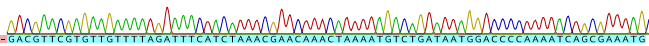

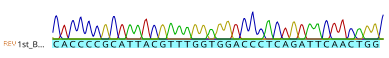

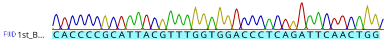

Supplementary Figure 1. Sanger sequences of BetaCoV/Singapore/12/2020 $\Delta 382$ mapped to Wuhan-Hu-1 showing the position of the 382-nt deletion in the SARS-CoV-2 genome. 
bioRxiv preprint doi: https://doi.org/10.1101/2020.03.11.987222; this version posted March 12, 2020. The copyright holder for this preprint (which was not certified by peer review) is the author/funder. All rights reserved. No reuse allowed without permission.

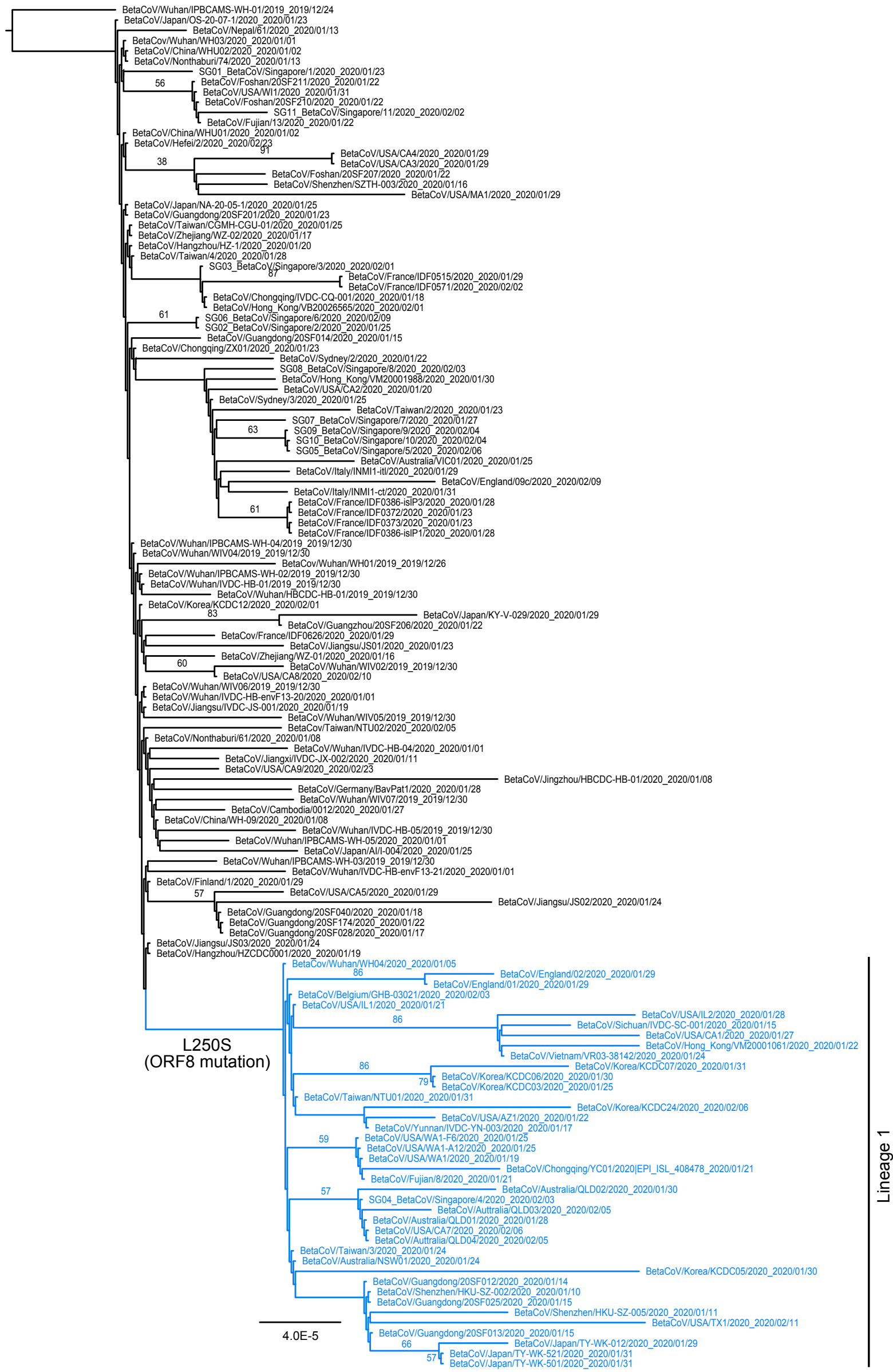

Supplementary Figure 2. Maximum-likelihood tree of SARS-CoV-2 genomes reconstructed using RAxML with 1,000 bootstrap replicates. $\triangle 382$ viruses were exlcluded from this analysis as the L250S mutation is located in the ORF8 deletion. 
Supplementary Table 1.

\begin{tabular}{|c|c|c|c|}
\hline Accession number & Virus isolate & Collection date & Note \\
\hline EPI_ISL_414378 & BetaCoV/Singapore/12/2020 & $17 / 02 / 2020$ & 382-nt deletion (this study) \\
\hline EPI_ISL_414379 & BetaCoV/Singapore/13/2020 & $18 / 02 / 2020$ & 382-nt deletion (this study) \\
\hline EPI_ISL_414380 & BetaCoV/Singapore/14/2020 & $19 / 02 / 2020$ & 382-nt deletion (this study) \\
\hline EPI_ISL_407987 & BetaCoV/Singapore/2/2020 & $25 / 01 / 2020$ & Wild type (this study) \\
\hline EPI_ISL_407988 & BetaCoV/Singapore/3/2020 & $01 / 02 / 2020$ & Wild type (this study) \\
\hline EPI_ISL_410535 & BetaCoV/Singapore/4/2020 & $03 / 02 / 2020$ & Wild type (this study) \\
\hline EPI_ISL_410536 & BetaCoV/Singapore/5/2020 & $06 / 02 / 2020$ & Wild type (this study) \\
\hline EPI_ISL_410537 & BetaCoV/Singapore/6/2020 & $09 / 02 / 2020$ & Wild type (this study) \\
\hline EPI_ISL_402119 & BetaCoV/Wuhan/IVDC-HB-01/2019 & $30 / 12 / 2019$ & Wild type \\
\hline EPI_ISL_402120 & BetaCoV/Wuhan/IVDC-HB-04/2020 & $01 / 01 / 2020$ & Wild type \\
\hline EPI_ISL_402121 & BetaCoV/Wuhan/IVDC-HB-05/2019 & $30 / 12 / 2019$ & Wild type \\
\hline EPI_ISL_402123 & BetaCoV/Wuhan/IPBCAMS-WH-01/2019 & $24 / 12 / 2019$ & Wild type \\
\hline EPI_ISL_402124 & BetaCoV/Wuhan/WIV04/2019 & $30 / 12 / 2019$ & Wild type \\
\hline EPI_ISL_402127 & BetaCoV/Wuhan/WIV02/2019 & $30 / 12 / 2019$ & Wild type \\
\hline EPI_ISL_402128 & BetaCoV/Wuhan/WIV05/2019 & $30 / 12 / 2019$ & Wild type \\
\hline EPI_ISL_402129 & BetaCoV/Wuhan/WIV06/2019 & $30 / 12 / 2019$ & Wild type \\
\hline EPI_ISL_402130 & BetaCoV/Wuhan/WIV07/2019 & $30 / 12 / 2019$ & Wild type \\
\hline EPI_ISL_402132 & BetaCoV/Wuhan/HBCDC-HB-01/2019 & $30 / 12 / 2019$ & Wild type \\
\hline EPI_ISL_403928 & BetaCoV/Wuhan/IPBCAMS-WH-05/2020 & $01 / 01 / 2020$ & Wild type \\
\hline EPI_ISL_403929 & BetaCoV/Wuhan/IPBCAMS-WH-04/2019 & $30 / 12 / 2019$ & Wild type \\
\hline EPI_ISL_403930 & BetaCoV/Wuhan/IPBCAMS-WH-03/2019 & $30 / 12 / 2019$ & Wild type \\
\hline EPI_ISL_403931 & BetaCoV/Wuhan/IPBCAMS-WH-02/2019 & $30 / 12 / 2019$ & Wild type \\
\hline EPI_ISL_403932 & BetaCoV/Guangdong/20SF012/2020 & $14 / 01 / 2020$ & Wild type \\
\hline EPI_ISL_403933 & BetaCoV/Guangdong/20SF013/2020 & $15 / 01 / 2020$ & Wild type \\
\hline EPI_ISL_403934 & BetaCoV/Guangdong/20SF014/2020 & $15 / 01 / 2020$ & Wild type \\
\hline EPI_ISL_403935 & BetaCoV/Guangdong/20SF025/2020 & $15 / 01 / 2020$ & Wild type \\
\hline EPI_ISL_403936 & BetaCoV/Guangdong/20SF028/2020 & $17 / 01 / 2020$ & Wild type \\
\hline EPI_ISL_403937 & BetaCoV/Guangdong/20SF040/2020 & $18 / 01 / 2020$ & Wild type \\
\hline EPI_ISL_403962 & BetaCoV/Nonthaburi/61/2020 & $08 / 01 / 2020$ & Wild type \\
\hline EPI_ISL_403963 & BetaCoV/Nonthaburi/74/2020 & $13 / 01 / 2020$ & Wild type \\
\hline EPI_ISL_404227 & BetaCoV/Zhejiang/WZ-01/2020 & $16 / 01 / 2020$ & Wild type \\
\hline EPI_ISL_404228 & BetaCoV/Zhejiang/WZ-02/2020 & $17 / 01 / 2020$ & Wild type \\
\hline EPI_ISL_404253 & BetaCoV/USA/IL1/2020 & $21 / 01 / 2020$ & Wild type \\
\hline EPI_ISL_404895 & BetaCoV/USA/WA1/2020 & $19 / 01 / 2020$ & Wild type \\
\hline EPI_ISL_405839 & BetaCoV/Shenzhen/HKU-SZ-005/2020 & $11 / 01 / 2020$ & Wild type \\
\hline EPI_ISL_406030 & BetaCoV/Shenzhen/HKU-SZ-002/2020 & $10 / 01 / 2020$ & Wild type \\
\hline EPI_ISL_406031 & BetaCoV/Taiwan/2/2020 & $23 / 01 / 2020$ & Wild type \\
\hline EPI_ISL_406034 & BetaCoV/USA/CA1/2020 & $27 / 01 / 2020$ & Wild type \\
\hline EPI_ISL_406036 & BetaCoV/USA/CA2/2020 & $20 / 01 / 2020$ & Wild type \\
\hline EPI_ISL_406223 & BetaCoV/USA/AZ1/2020 & $22 / 01 / 2020$ & Wild type \\
\hline EPI_ISL_406531 & BetaCoV/Guangdong/20SF174/2020 & $22 / 01 / 2020$ & Wild type \\
\hline EPI_ISL_406533 & BetaCoV/Guangzhou/20SF206/2020 & $22 / 01 / 2020$ & Wild type \\
\hline EPI_ISL_406534 & BetaCoV/Foshan/20SF207/2020 & $22 / 01 / 2020$ & Wild type \\
\hline EPI_ISL_406535 & BetaCoV/Foshan/20SF210/2020 & $22 / 01 / 2020$ & Wild type \\
\hline EPI_ISL_406536 & BetaCoV/Foshan/20SF211/2020 & $22 / 01 / 2020$ & Wild type \\
\hline EPI_ISL_406538 & BetaCoV/Guangdong/20SF201/2020 & $23 / 01 / 2020$ & Wild type \\
\hline EPI_ISL_406594 & BetaCoV/Shenzhen/SZTH-003/2020 & $16 / 01 / 2020$ & Wild type \\
\hline EPI_ISL_406597 & BetaCoV/France/IDF0373/2020 & $23 / 01 / 2020$ & Wild type \\
\hline EPI_ISL_406716 & BetaCoV/China/WHU01/2020 & $02 / 01 / 2020$ & Wild type \\
\hline EPI_ISL_406717 & BetaCoV/China/WHU02/2020 & $02 / 01 / 2020$ & Wild type \\
\hline EPI_ISL_406798 & BetaCov/Wuhan/WH01/2019 & $26 / 12 / 2019$ & Wild type \\
\hline EPI_ISL_406800 & BetaCov/Wuhan/WH03/2020 & $01 / 01 / 2020$ & Wild type \\
\hline EPI_ISL_406801 & BetaCov/Wuhan/WH04/2020 & $05 / 01 / 2020$ & Wild type \\
\hline EPI_ISL_406844 & BetaCoV/Australia/VIC01/2020 & $25 / 01 / 2020$ & Wild type \\
\hline EPI_ISL_406862 & BetaCoV/Germany/BavPat1/2020 & $28 / 01 / 2020$ & Wild type \\
\hline EPI_ISL_406970 & BetaCoV/Hangzhou/HZ-1/2020 & $20 / 01 / 2020$ & Wild type \\
\hline EPI_ISL_406973 & BetaCoV/Singapore/1/2020 & $23 / 01 / 2020$ & Wild type \\
\hline EPI ISL 407071 & BetaCoV/England/01/2020 & $29 / 01 / 2020$ & Wild type \\
\hline
\end{tabular}


EPI_ISL_407073 EPI_ISL_407079 EPI_ISL_407084 EPI_ISL_407193 EPI_ISL_407214 EPI_ISL_407215 EPI_ISL_407313 EPI_ISL_407893 EPI_ISL_407894 EPI_ISL_407896 EPI_ISL_407976 EPI_ISL_408008 EPI_ISL_408009 EPI_ISL_408010 EPI_ISL_408430 EPI_ISL_408431 EPI_ISL_408478 EPI_ISL_408479 EPI_ISL_408480 EPI_ISL_408481 EPI_ISL_408484 EPI_ISL_408486 EPI_ISL_408488 EPI_ISL_408489 EPI_ISL_408514 EPI_ISL_408515 EPI_ISL_408665 EPI_ISL_408666 EPI_ISL_408667 EPI_ISL_408668 EPI_ISL_408669 EPI_ISL_408670 EPI_ISL_408976 EPI_ISL_408977 EPI_ISL_409067 EPI_ISL_410045 EPI_ISL_410218 EPI_ISL_410301 EPI_ISL_410531 EPI_ISL_410532 EPI_ISL_410545 EPI_ISL_410546 EPI_ISL_410713 EPI_ISL_410714 EPI_ISL_410715 EPI_ISL_410716 EPI_ISL_410717 EPI_ISL_410718 EPI_ISL_410719 EPI_ISL_410720 EPI_ISL_411060 EPI_ISL_411066 EPI_ISL_411218 EPI_ISL_411219 EPI_ISL_411220 EPI_ISL_411902 EPI_ISL_411915 EPI_ISL_411926 EPI_ISL_411927 EPI_ISL_411950 EPI_ISL_411952
BetaCoV/England/02/2020

BetaCoV/Finland/1/2020

BetaCoV/Japan/AI/I-004/2020

BetaCoV/Korea/KCDC03/2020

BetaCoV/USA/WA1-A12/2020

BetaCoV/USA/WA1-F6/2020

BetaCoV/Hangzhou/HZCDC0001/2020

BetaCoV/Australia/NSW01/2020

BetaCoV/Australia/QLD01/2020

BetaCoV/Australia/QLD02/2020

BetaCoV/Belgium/GHB-03021/2020

BetaCoV/USA/CA3/2020

BetaCoV/USA/CA4/2020

BetaCoV/USA/CA5/2020

BetaCoV/France/IDF0515/2020

BetaCov/France/IDF0626/2020

BetaCoV/Chongqing/YC01/2020

BetaCoV/Chongqing/ZX01/2020

BetaCoV/Yunnan/IVDC-YN-003/2020

BetaCoV/Chongqing/IVDC-CQ-001/2020

BetaCoV/Sichuan/IVDC-SC-001/2020

BetaCoV/Jiangxi/IVDC-JX-002/2020

BetaCoV/Jiangsu/IVDC-JS-001/2020

BetaCoV/Taiwan/NTU01/2020

BetaCoV/Wuhan/IVDC-HB-envF13-20/2020

BetaCoV/Wuhan/IVDC-HB-envF13-21/2020

BetaCoV/Japan/TY-WK-012/2020

BetaCoV/Japan/TY-WK-501/2020

BetaCoV/Japan/TY-WK-521/2020

BetaCoV/Vietnam/VR03-38142/2020

BetaCoV/Japan/KY-V-029/2020

BetaCoV/USA/WI1/2020

BetaCoV/Sydney/2/2020

BetaCoV/Sydney/3/2020

BetaCoV/USA/MA1/2020

BetaCoV/USA/IL2/2020

BetaCov/Taiwan/NTU02/2020

BetaCoV/Nepal/61/2020

BetaCoV/Japan/NA-20-05-1/2020

BetaCoV/Japan/OS-20-07-1/2020

BetaCoV/Italy/INMI1-itl/2020

BetaCoV/Italy/INMI1-ct/2020

BetaCoV/Singapore/7/2020

BetaCoV/Singapore/8/2020

BetaCoV/Singapore/9/2020

BetaCoV/Singapore/10/2020

BetaCoV/Australia/QLD03/2020

BetaCoV/Australia/QLD04/2020

BetaCoV/Singapore/11/2020

BetaCoV/France/IDF0372/2020

BetaCoV/Fujian/8/2020

BetaCoV/Fujian/13/2020

BetaCoV/France/IDF0571/2020

BetaCoV/France/IDF0386-isIP1/2020

BetaCoV/France/IDF0386-isIP3/2020

BetaCoV/Cambodia/0012/2020

BetaCoV/Taiwan/CGMH-CGU-01/2020

BetaCoV/Taiwan/3/2020

BetaCoV/Taiwan/4/2020

BetaCoV/Jiangsu/JS01/2020

BetaCoV/Jiangsu/JSO2/2020
$29 / 01 / 2020$

$29 / 01 / 2020$

$25 / 01 / 2020$

$25 / 01 / 2020$

$25 / 01 / 2020$

$25 / 01 / 2020$

$19 / 01 / 2020$

$24 / 01 / 2020$

$28 / 01 / 2020$

$30 / 01 / 2020$

$03 / 02 / 2020$

$29 / 01 / 2020$

$29 / 01 / 2020$

$29 / 01 / 2020$

$29 / 01 / 2020$

$29 / 01 / 2020$

$21 / 01 / 2020$

$23 / 01 / 2020$

$17 / 01 / 2020$

$18 / 01 / 2020$

$15 / 01 / 2020$

$11 / 01 / 2020$

$19 / 01 / 2020$

$31 / 01 / 2020$

$01 / 01 / 2020$

$01 / 01 / 2020$

$29 / 01 / 2020$

$31 / 01 / 2020$

$31 / 01 / 2020$

$24 / 01 / 2020$

$29 / 01 / 2020$

$31 / 01 / 2020$

$22 / 01 / 2020$

$25 / 01 / 2020$

$29 / 01 / 2020$

$28 / 01 / 2020$

$05 / 02 / 2020$

$13 / 01 / 2020$

$25 / 01 / 2020$

$23 / 01 / 2020$

$29 / 01 / 2020$

$31 / 01 / 2020$

$27 / 01 / 2020$

$03 / 02 / 2020$

$04 / 02 / 2020$

$04 / 02 / 2020$

$05 / 02 / 2020$

$05 / 02 / 2020$

$02 / 02 / 2020$

$23 / 01 / 2020$

$21 / 01 / 2020$

$22 / 01 / 2020$

$02 / 02 / 2020$

$28 / 01 / 2020$

$28 / 01 / 2020$

$27 / 01 / 2020$

$25 / 01 / 2020$

$24 / 01 / 2020$

$28 / 01 / 2020$

$23 / 01 / 2020$

$24 / 01 / 2020$
Wild type

Wild type

Wild type

Wild type

Wild type

Wild type

Wild type

Wild type

Wild type

Wild type

Wild type

Wild type

Wild type

Wild type

Wild type

Wild type

Wild type

Wild type

Wild type

Wild type

Wild type

Wild type

Wild type

Wild type

Wild type

Wild type

Wild type

Wild type

Wild type

Wild type

Wild type

Wild type

Wild type

Wild type

Wild type

Wild type

Wild type

Wild type

Wild type

Wild type

Wild type

Wild type

Wild type

Wild type

Wild type

Wild type

Wild type

Wild type

Wild type

Wild type

Wild type

Wild type

Wild type

Wild type

Wild type

Wild type

Wild type

Wild type

Wild type

Wild type

Wild type 
bioRxiv preprint doi: https://doi.org/10.1101/2020.03.11.987222; this version posted March 12, 2020. The copyright holder for this preprint (which was not certified by peer review) is the author/funder. All rights reserved. No reuse allowed without permission.

EPI_ISL_411953
EPI_ISL_411954
EPI_ISL_411955
EPI_ISL_411956
EPI_ISL_411957
EPI_ISL_412026
EPI_ISL_412028
EPI_ISL_412029
EPI_ISL_412030
EPI_ISL_412116
EPI_ISL_412459
EPI_ISL_412862
EPI_ISL_412869
EPI_ISL_412870
EPI_ISL_412871
EPI_ISL_412872
EPI_ISL_412873

EPI_ISL_411953

EPI ISL 411955

EPI_ISL_411956

EPIISL 412026

EPI_ISL_412028

EPI_ISL_412029

EPI_ISL_412030

EPI_ISL_412459

EPI_ISL_412862

EPI_ISL_412869

EPI_ISL_412870

EPI_ISL_412872

EPI_ISL_412873
BetaCoV/Jiangsu/JS03/2020

BetaCoV/USA/CA7/2020

BetaCoV/USA/CA8/2020

BetaCoV/USA/TX1/2020

BetaCoV/China/WH-09/2020

BetaCoV/Hefei/2/2020

BetaCoV/Hong_Kong/VM20001061/2020

BetaCoV/Hong_Kong/VM20001988/2020

BetaCoV/Hong_Kong/VB20026565/2020

BetaCoV/England/09c/2020

BetaCoV/Jingzhou/HBCDC-HB-01/2020

BetaCoV/USA/CA9/2020

BetaCoV/Korea/KCDC05/2020

BetaCoV/Korea/KCDC06/2020

BetaCoV/Korea/KCDC07/2020

BetaCoV/Korea/KCDC12/2020

BetaCoV/Korea/KCDC24/2020
$24 / 01 / 2020$

$06 / 02 / 2020$

$10 / 02 / 2020$

$11 / 02 / 2020$

$08 / 01 / 2020$

$23 / 02 / 2020$

$22 / 01 / 2020$

$30 / 01 / 2020$

$01 / 02 / 2020$

$09 / 02 / 2020$

$08 / 01 / 2020$

$23 / 02 / 2020$

$30 / 01 / 2020$

$30 / 01 / 2020$

$31 / 01 / 2020$

$01 / 02 / 2020$

$06 / 02 / 2020$
Wild type

Wild type

Wild type

Wild type

Wild type

Wild type

Wild type

Wild type

Wild type

Wild type

Wild type

Wild type

Wild type

Wild type

Wild type

Wild type

Wild type 